IMA Journal of Numerical Analysis (2013) 33, 1156-1175

doi:10.1093/imanum/drs034

Advance Access publication on January 15, 2013

\title{
Generalized convolution quadrature with variable time stepping
}

\author{
Maria Lopez-Fernandez* and Stefan Sauter \\ Institut für Mathematik, Universität Zürich, Winterthurerstrasse 190, CH-8057 Zürich, Switzerland \\ *Corresponding author: maria.lopez@math.uzh.ch stas@math.uzh.ch
}

[Received on 21 December 2011; revised on 27 August 2012]

\begin{abstract}
In this paper, we will present a generalized convolution quadrature for solving linear parabolic and hyperbolic evolution equations. The original convolution quadrature method by Lubich works very nicely for equidistant time steps while the generalization of the method and its analysis to nonuniform time stepping is by no means obvious. We will introduce the generalized convolution quadrature allowing for variable time steps and develop a theory for its error analysis. This method opens the door for further development towards adaptive time stepping for evolution equations. As the main application of our new theory, we will consider the wave equation in exterior domains which is formulated as a retarded boundary integral equation.
\end{abstract}

Keywords: variable step size; convolution quadrature; convolution equations; retarded potentials; boundary integral equations; wave equation.

\section{Introduction}

In this paper, we will present a numerical method for the discretization of linear convolution equations of the form

$$
k * \phi=g
$$

where $*$ denotes convolution with respect to time, $g$ is a given function and $k$ is some fixed kernel function, in such a way that the left-hand side in (1.1) is understood as a mapping of the function $\phi$ into some function space. In many applications such as partial differential equations of hyperbolic or parabolic type, the kernel function $k$ is defined as the inverse Laplace transform of the transfer function $\mathcal{K}$ in the Laplace domain and analyticity of $\mathcal{K}$ is assumed in a region containing the half plane $\operatorname{Re} z \geqslant \sigma_{0}>0$. For this type of problem, the convolution quadrature method was developed originally by Lubich; see Lubich (1988a,b), Lubich \& Ostermann (1993) and Lubich (2004) for parabolic problems and Lubich (1994) for hyperbolic ones. The idea is to express the convolution kernel $k$ as the inverse Laplace transform of $\mathcal{K}$ and reduce the problem to the solution of scalar ordinary differential equations (ODEs) of the form $y^{\prime}=z y+g$, for $z$ the variable in the Laplace domain. The temporal discretization is then based on the approximation of the solution of these ODEs by some time-stepping method and the transformation of the resulting equation back to the original time domain. This results in a discrete convolution in time which has very nice properties: (a) it allows for Fast Fourier-type algorithms for solving the discrete convolution equation and (b) the theory of ODEs can be employed nicely for deriving error estimates in the Laplace domain, and then these estimates can be transformed back to the original time domain via Parseval's theorem.

On the other hand, there is also a drawback in the convolution quadrature method. Since it heavily employs the continuous and discrete Fourier-Laplace transforms for the formulation of the method and 
its analysis, the generalization to variable time stepping is by no means obvious. However, if the righthand side is not uniformly smooth and/or contains nonuniformly distributed variations in time and/or consists of localized pulses, the use of adaptive time stepping becomes very important in order to keep the number of time steps reasonably small. Furthermore, the introduction of adaptivity is the first step in the development of strategies to control the step size in terms of the behaviour of the solution to the integral equation.

In this paper, we will present a generalized convolution quadrature which allows for variable time stepping and develop a new theory for its error analysis. We limit ourselves to the implicit Euler method for the time discretization. Note that the use of low-order methods is justified for problems where the solution, possibly, contains nonuniformly distributed irregularities. We emphasize that our derivation of the method can be extended to higher-order Runge-Kutta methods, but the representation of the discrete solution becomes more complicated and the extension of the analysis is by no means straightforward. It is our opinion that fully understanding the first-order method will open the way to further developments, both from the analytical and the algorithmic point of view.

Our idea is based on introducing adaptivity in the time integration of the scalar ODEs $y^{\prime}=$ $z y+g$. This idea has already been used in the oblivious algorithm which was developed in Lopez-Fernandez et al. (2008). However, this algorithm is restricted to sectorial convolution kernels, for which the analyticity domain of $\mathcal{K}$ extends to a sector entering the left half plane (see Lopez-Fernandez et al., 2008 for details) and, thus, is not applicable to wave equations. Furthermore, no error analysis is available. Our main application is the solution of retarded potential integral equations (RPIE) which arise if the wave equation in an unbounded exterior domain is formulated as a space-time integral equation on the boundary of the scatterer. The most popular numerical approaches for its discretization in the literature are (a) the direct space-time Galerkin discretization of the RPIE and (b) the convolution quadrature. For the first class of methods, only very recently a temporal discretization with variable time steps has been proposed (Sauter \& Veit, 2012), while for the convolution quadrature, to the best of our knowledge, such a generalization does not exist in the literature.

The paper is organized as follows. In Section 2, we introduce abstract one-sided convolution equations and formulate appropriate assumptions on the growth behaviour of the transfer operator in some complex half plane. Section 3 is concerned with the temporal discretization of the convolution equation via the time integration of a parameter-dependent ODE in the Laplace domain. The error analysis for the discretization of this abstract equation is developed in Section 4. We apply this theory to the space-time discretization of RPIE for solving the wave equation in Section 5 and give some concluding remarks in Section 6. Some technical estimates for the stability function for the implicit Euler method are postponed to the Appendix.

The efficient implementation of the method presented in this paper is challenging. In Lopez-Fernandez \& Sauter (2012b) a fully discrete algorithmic version of our method is presented, which allows the integral operators in the arising time-stepping scheme to be computed in a fast and stable way. In this paper, the results of numerical experiments are also reported which illustrate the advantage of variable time stepping for some important classes of applications.

\section{One-sided convolution equations}

We consider the class of convolution operators as described in Lubich (1994, Section 2.1) and recall its definition. Let $B$ and $D$ denote some normed vector spaces and let $\mathcal{L}(B, D)$ be the space of continuous, 
linear mappings. As a norm in $\mathcal{L}(B, D)$, we take the usual operator norm

$$
\|\mathcal{F}\|:=\sup _{u \in B \backslash\{0\}} \frac{\|\mathcal{F} u\|_{D}}{\|u\|_{B}} .
$$

For a given right-hand side $g: \mathbb{R}_{\geqslant 0} \rightarrow D$, we consider the problem of finding $\phi: \mathbb{R}_{\geqslant 0} \rightarrow B$ such that for all $t \geqslant 0$,

$$
\int_{0}^{t} k(t-\tau) \phi(\tau) \mathrm{d} \tau=g(t)
$$

is considered as an equation in $D$. The kernel operator $k$ is the inverse Laplace transform of some transfer operator $\mathcal{K}$, which is defined as follows. Let $\mathcal{K}: I_{\sigma_{0}} \rightarrow \mathcal{L}(B, D)$ be an analytic operator-valued function in a half plane

$$
I_{\sigma_{0}}:=\left\{z \in \mathbb{C}: \operatorname{Re} z \geqslant \sigma_{0}\right\}, \quad \text { for some } \sigma_{0}>0,
$$

which is bounded by

$$
\|\mathcal{K}(z)\| \leqslant C_{\text {op }}|z|^{\theta} \quad \forall z \in I_{\sigma_{0}},
$$

for some $C_{\text {op }}>0$ and $\theta \in \mathbb{R}$. For $\rho \in \mathbb{Z}$, we define

$$
\mathcal{K}_{\rho}(z):=z^{-\rho} \mathcal{K}(z)
$$

We choose $\rho>\max \{-1, \theta+1\}$, so that the Laplace inversion formula

$$
k_{\rho}(t):=\frac{1}{2 \pi \mathrm{i}} \int_{\gamma} e^{z t} \mathcal{K}_{\rho}(z) \mathrm{d} z
$$

for a contour $\gamma=\sigma+\mathrm{i} \mathbb{R}, \sigma \geqslant \sigma_{0}$ defines a continuous and exponentially bounded operator $k_{\rho}(t)$, which by Cauchy's integral theorem vanishes for $t<0$. As in Lubich (1994), we denote the convolution $k * \phi$ by

$$
\left(\mathcal{K}\left(\partial_{t}\right) \phi\right)(t):=\left(\frac{\mathrm{d}}{\mathrm{d} t}\right)^{\rho} \int_{-\infty}^{t} k_{\rho}(t-\tau) \phi(\tau) \mathrm{d} \tau=\int_{0}^{\infty} k_{\rho}(\tau) \phi^{(\rho)}(t-\tau) \mathrm{d} \tau,
$$

for sufficiently smooth functions $\phi$ which satisfy $\phi(x)=0$ for $x \leqslant 0$.

Our goal is to solve the convolution equation

$$
\mathcal{K}\left(\partial_{t}\right) \phi=g,
$$

where we always assume that the given right-hand side can be extended by zero to $t \leqslant 0$ to a sufficiently smooth function. Additional smoothness assumptions at $t=0$ will be formulated later.

The composition rule for one-sided convolutions (cf. Lubich, 1994, (2.3), (2.22)) leads to

$$
\phi=\mathcal{K}^{-1}\left(\partial_{t}\right) g
$$

so that

$$
\phi(t)=\int_{0}^{t}\left(\frac{1}{2 \pi \mathrm{i}} \int_{\gamma} e^{z \tau}\left(\mathcal{K}^{-1}\right)_{\rho}(z) \mathrm{d} z\right) g^{(\rho)}(t-\tau) \mathrm{d} \tau,
$$

for appropriately chosen $\rho$. This representation of the solution clearly shows that the growth behaviour of $\left\|\mathcal{K}^{-1}(z)\right\|$ determines the smoothness requirements on the right-hand side $g$. We will assume that, for 
some $C_{\text {op }}>0$ and $\mu \in \mathbb{R}$, a similar estimate ${ }^{1}$ to (2.3) holds for $\mathcal{K}^{-1}$, namely,

$$
\left\|\mathcal{K}^{-1}(z)\right\| \leqslant C_{\text {op }}|z|^{\mu} \quad \forall z \in I_{\sigma_{0}} .
$$

In this way, $\rho$ will be chosen according to

$$
\rho>\max \{-1, \mu+1, \theta+1\}
$$

\section{Temporal discretization}

Our main application will be the time discretization of retarded potentials associated to wave equations. In this context, (2.9) will typically hold for some $\mu>0$. As we have seen in the previous section, solving the convolution equation (2.7) is equivalent to the evaluation of the convolution with the inverse transfer function applied to the right-hand side (cf. (2.8)), which can be written in compact form as

$$
\phi=\left(\mathcal{K}^{-1}\right)_{\rho}\left(\partial_{t}\right) g^{(\rho)} .
$$

In an abstract setting, we are concerned with the approximation of the mapping

$$
f \mapsto \mathcal{C}\left(\partial_{t}\right) f
$$

at prescribed time points for a given transfer operator $\mathcal{C}$ which satisfies a bound like (2.9) for some $\mu<-1$. To discretize (3.1), we express the mapping in (3.1) as the inverse Laplace transform of the transfer operator

$$
\left(\mathcal{C}\left(\partial_{t}\right) f\right)(t)=\frac{1}{2 \pi \mathrm{i}} \int_{0}^{t}\left(\int_{\gamma} e^{z(t-\tau)} \mathcal{C}(z) \mathrm{d} z\right) f(\tau) \mathrm{d} \tau .
$$

By interchanging the order of integration, we can write

$$
\left(\mathcal{C}\left(\partial_{t}\right) f\right)(t)=\frac{1}{2 \pi \mathrm{i}} \int_{\gamma} \mathcal{C}(z) u(z, t) \mathrm{d} z
$$

where

$$
u(z, t):=\int_{0}^{t} e^{z(t-\tau)} f(\tau) \mathrm{d} \tau .
$$

Note that the function $u$ in (3.2) is the solution of the initial value problem

$$
u_{t}(z, t)=z u(z, t)+f(t), \quad u(z, 0)=0 .
$$

We will consider the implicit Euler method with variable mesh width for the discretization of (3.3). For a time mesh $t_{0}=0<t_{1}<t_{2}<\cdots<t_{N}=T$ with variable step sizes $\Delta_{j}=t_{j}-t_{j-1}, j=1, \ldots, N$, the

\footnotetext{
${ }^{1}$ For simplicity, we use the same notation for the multiplicative constants in (2.3) and (2.9).
} 
implicit Euler method for (3.3) is given by

$$
u_{n}(z)=\frac{1}{1-\Delta_{n} z} u_{n-1}(z)+\frac{\Delta_{n}}{1-\Delta_{n} z} f_{n}, \quad u_{0}(z)=0 .
$$

This recursion can be resolved and we obtain

$$
u\left(z, t_{n}\right) \approx u_{n}(z)=\sum_{j=1}^{n} \Delta_{j} f_{j} \prod_{k=j}^{n} \frac{1}{1-\Delta_{k} z} .
$$

By considering (3.2) at time point $t_{n}$ and replacing $u\left(z, t_{n}\right)$ by the approximation $u_{n}(z)$, we obtain the approximation to the convolution $\left(\mathcal{C}\left(\partial_{t}\right) f\right)\left(t_{n}\right)$,

$$
\left(\mathcal{C}\left(\partial_{t}\right) f\right)\left(t_{n}\right) \approx \frac{1}{2 \pi \mathrm{i}} \int_{\gamma} \mathcal{C}(z) u_{n}(z) \mathrm{d} z
$$

By solving the recursion (3.4) and using Cauchy's integral formula for the divided differences of an analytic function (see for instance de Boor, 2005, Formula (51)), we conclude from the combination of (3.5) and (3.6) that the approximation in (3.6) can be written in the form

$$
\left(\mathcal{C}\left(\partial_{t}\right) f\right)\left(t_{n}\right) \approx \sum_{j=1}^{n} \omega_{n, j}(0)\left(\left[\frac{1}{\Delta_{j}}, \frac{1}{\Delta_{j+1}}, \ldots, \frac{1}{\Delta_{n}}\right] \mathcal{C}\right) f_{j},
$$

where $\omega_{n, j}(z)=\prod_{\ell=j+1}^{n}\left(z-\Delta_{\ell}^{-1}\right)$ and $\left[1 / \Delta_{j}, 1 / \Delta_{j+1}, \ldots, 1 / \Delta_{n}\right] \mathcal{C}$ denote Newton's divided difference with respect to the nodes $\Delta_{k}^{-1}, j \leqslant k \leqslant n$.

For $\mathcal{C}=\left(\mathcal{K}^{-1}\right)_{\rho}$ and $f=g^{(\rho)}$, formula (3.7) defines an approximation $\phi_{n} \approx \phi\left(t_{n}\right)$ to the solution of the convolution equation (2.7). In order to define a recursive method based on $\mathcal{K}$ rather than $\mathcal{K}^{-1}$, we need the following lemma.

Lemma 3.1 (Inversion formula) Let $\left(x_{i}\right)_{i \in \mathbb{N}} \subset \mathbb{R}$ denote a sequence of points and let $f: \mathbb{R} \rightarrow E$ denote some function in a set $E$ of linear mappings. We assume that $f\left(x_{i}\right)$ is invertible for all $i \in \mathbb{N}_{\geqslant 1}$. Let $\tilde{\omega}_{n, j}(x):=\prod_{\ell=j+1}^{n}\left(x-x_{\ell}\right)$. A mapping of the form

$$
q_{n}:=\sum_{j=1}^{n} \tilde{\omega}_{n, j}(0)\left(\left[x_{j}, x_{j+1}, \ldots, x_{n}\right] f\right) u_{j}, \quad n \in \mathbb{N}_{\geqslant 1}
$$

can be inverted and it holds that

$$
u_{n}=\sum_{j=1}^{n} \tilde{\omega}_{n, j}(0)\left(\left[x_{j}, x_{j+1}, \ldots, x_{n}\right] f^{-1}\right) q_{j}, \quad n \in \mathbb{N}_{\geqslant 1} .
$$

Proof. We denote the left-hand side in (3.9) by $\tilde{u}_{n}$ and prove that $\tilde{u}_{n}=u_{n}$ if we replace $q_{j}$ by the definition (3.8). By inserting (3.8) into the right-hand side of (3.9), we obtain

$$
\tilde{u}_{n}=\sum_{j=1}^{n} \tilde{\omega}_{n, j}(0)\left[x_{j}, x_{j+1}, \ldots, x_{n}\right] f^{-1} \sum_{k=1}^{j} \tilde{\omega}_{j, k}(0)\left(\left[x_{k}, x_{k+1}, \ldots, x_{j}\right] f\right) u_{k} .
$$


Observe that $\tilde{\omega}_{n, j}(0) \tilde{\omega}_{j, k}(0)=\tilde{\omega}_{n, k}(0)$, so that after interchanging the ordering of the summations,

$$
\tilde{u}_{n}=\sum_{k=1}^{n} \tilde{\omega}_{n, k}(0)\left(\sum_{j=k}^{n}\left(\left[x_{j}, x_{j+1}, \ldots, x_{n}\right] f^{-1}\right)\left(\left[x_{k}, x_{k+1}, \ldots, x_{j}\right] f\right)\right) u_{k} .
$$

The Leibniz rule for divided differences (de Boor, 2005, Corollary 28) leads to

$$
\begin{aligned}
\sum_{j=k}^{n}\left(\left[x_{j}, x_{j+1}, \ldots, x_{n}\right] f^{-1}\right)\left(\left[x_{k}, x_{k+1}, \ldots, x_{j}\right] f\right) & =\left[x_{k}, x_{k+1}, \ldots, x_{n}\right]\left(f^{-1} f\right) \\
& =\left[x_{k}, x_{k+1}, \ldots, x_{n}\right] I=\delta_{k, n} I .
\end{aligned}
$$

Here, $I$ is the identity mapping considered as a constant function in $x$. Hence, only the summand with $k=n$ in (3.10) is different from zero and the assertion follows.

DEFINITION 3.2 (Generalized convolution quadrature) For a set of given time points $\left(t_{i}\right)_{i=1}^{N}$ the generalized convolution quadrature (GCQ) approximation of

$$
\mathcal{K}\left(\partial_{t}\right) \phi=g
$$

is given by

$$
\mathcal{K}_{-\rho}\left(\frac{1}{\Delta_{n}}\right) \phi_{n}=g_{n}^{(\rho)}-\sum_{j=1}^{n-1} \omega_{n, j}(0)\left(\left[\frac{1}{\Delta_{j}}, \frac{1}{\Delta_{j+1}}, \ldots, \frac{1}{\Delta_{n}}\right] \mathcal{K}_{-\rho}\right) \phi_{j}
$$

where $\mathcal{K}_{-\rho}(z):=z^{\rho} \mathcal{K}(z)$; cf. (2.4).

REMARK 3.3 The representation of the GCQ in the form (3.11) is well suited for theoretical investigations but not for practical implementation due to numerical instabilities. In Lopez-Fernandez \& Sauter (2012b), an efficient, fully discrete algorithm of the GCQ is presented which is based on the representation of the high-order divided differences in (3.11) as contour integrals and their approximation by a novel contour quadrature method which has been developed in Lopez-Fernandez \& Sauter (2012a). For the details and the results of numerical experiments, we refer to Lopez-Fernandez \& Sauter (2012b).

\section{Error analysis}

For some fixed $N \geqslant 1$, we consider the discrete approximations $\phi_{n}$ defined by (3.11), for $1 \leqslant n \leqslant N$. We set

$$
\Delta:=\max \left\{\Delta_{j}: 1 \leqslant j \leqslant N\right\}
$$

From (3.11), we are interested in the solution of the equation

$$
\sum_{j=1}^{n} \omega_{n, j}(0)\left(\left[\frac{1}{\Delta_{j}}, \frac{1}{\Delta_{j+1}}, \ldots, \frac{1}{\Delta_{n}}\right] \mathcal{K}_{-\rho}\right) \phi_{j}=g_{n}^{(\rho)}, \quad 1 \leqslant n \leqslant N .
$$




\subsection{Discrete stability}

Lemma 4.1 (Summation by parts) Let $\phi_{n}$ be the solution of (4.1). Assume that $g \in C^{\rho}([0, T])$ and it is extended by 0 to $t \leqslant 0$. Then $\phi_{n}$ has the representation

$$
\phi_{n}=\sum_{j=1}^{n}\left(\Delta_{j}+\Delta_{j-1}\right) Q_{j}^{(\rho+2, n)}\left[t_{j-2}, t_{j-1}, t_{j}\right] g^{(\rho)},
$$

where $t_{-1}<0$ can be chosen arbitrarily, ${ }^{2} \Delta_{0}:=t_{0}-t_{-1}$, and

$$
Q_{j}^{(k, n)}:=\frac{1}{2 \pi \mathrm{i}} \int_{\gamma} \frac{\mathcal{K}^{-1}(z)}{z^{k} \prod_{\ell=j}^{n}\left(1-\Delta_{\ell} z\right)} \mathrm{d} z \quad \forall 1 \leqslant j \leqslant n .
$$

Proof. As a consequence of Lemma 3.1, we obtain from (4.1),

$$
\begin{aligned}
\phi_{n} & =\sum_{j=1}^{n} \omega_{n, j}(0)\left(\left[\frac{1}{\Delta_{j}}, \frac{1}{\Delta_{j+1}}, \ldots, \frac{1}{\Delta_{n}}\right]\left(\mathcal{K}^{-1}\right)_{\rho}\right) g_{j}^{(\rho)} \\
& =\left(\mathcal{K}^{-1}\right)_{\rho}\left(\frac{1}{\Delta_{n}}\right) g_{n}^{(\rho)}+\sum_{j=1}^{n-1} \Delta_{j} Q_{j}^{(\rho, n)} g_{j}^{(\rho)} .
\end{aligned}
$$

Twice applying the relation

$$
\Delta_{j} Q_{j}^{(k, n)}=-\left(Q_{j+1}^{(k+1, n)}-Q_{j}^{(k+1, n)}\right),
$$

which can be verified straightforwardly, we obtain from (4.4) and following the notation ${ }^{3}$ in (2.4),

$$
\begin{aligned}
\phi_{n}= & \left(\mathcal{K}^{-1}\right)_{\rho}\left(\frac{1}{\Delta_{n}}\right) g_{n}^{(\rho)}-Q_{n}^{(\rho+1, n)} g_{n-1}^{(\rho)}-Q_{n}^{(\rho+2, n)}\left[t_{n-2}, t_{n-1}\right] g^{(\rho)} \\
& +\sum_{j=1}^{n-1}\left(\Delta_{j}+\Delta_{j-1}\right) Q_{j}^{(\rho+2, n)}\left[t_{j-2}, t_{j-1}, t_{j}\right] g^{(\rho)} .
\end{aligned}
$$

Using the relation $Q_{n}^{(k, n)}=\left(\mathcal{K}^{-1}\right)_{k-1}\left(1 / \Delta_{n}\right)$, we obtain

$$
\phi_{n}=\sum_{j=1}^{n}\left(\Delta_{j}+\Delta_{j-1}\right) Q_{j}^{(\rho+2, n)}\left[t_{j-2}, t_{j-1}, t_{j}\right] g^{(\rho)} .
$$

We next estimate the operators in the representation formula (4.2) for $\phi$.

Assumption 4.2 For all $\sigma_{0}>0$ and $z \in I_{\sigma_{0}}$, the transfer operator $\mathcal{K}(z): B \rightarrow D$ is invertible and for some $\mu \in \mathbb{R}$ satisfies the bound

$$
\left\|\mathcal{K}^{-1}(z)\right\| \leqslant C_{\text {op }}|z|^{\mu} \quad \forall z \in I_{\sigma_{0}},
$$

where $C_{\text {op }}$ depends on $\sigma_{0}$.

\footnotetext{
${ }^{2}$ For simplicity, we fix $t_{-1}:=-t_{1}$.

${ }^{3}$ Note that $\left(\mathcal{K}^{-1}\right)_{m}(z)$ is understood as $z^{-m} \mathcal{K}^{-1}(z)$ and not as $\left(\mathcal{K}_{m}(z)\right)^{-1}$.
} 
Lemma 4.3 Let Assumption 4.2 be satisfied and let $\Delta$ be sufficiently small that $1-\Delta \sigma_{0} \geqslant \alpha_{0}$ for some $\alpha_{0}>0$. Let $\rho$ be chosen according to (2.10). Then, there exists a constant $C$, depending only on $\sigma_{0}, \alpha_{0}$ and on the transfer operator $\mathcal{K}^{-1}$ through the constants $C_{\mathrm{op}}$ and $\mu$ in Assumption 4.2, such that for all $g \in D$ it holds that

$$
\left\|Q_{j}^{(\rho+2, n)} g\right\|_{B} \leqslant C e^{\delta_{0}\left(t_{n}-t_{j-1}\right)}\|g\|_{D} \quad \text { with } \delta_{0}:=\frac{\sigma_{0}}{1-\Delta \sigma_{0}} .
$$

Proof. From Assumption 4.2, we conclude that

$$
\begin{aligned}
\left\|Q_{j}^{(\rho+2, n)} g\right\|_{B} & \leqslant \frac{1}{2 \pi} \frac{1}{\prod_{\ell=j}^{n}\left|1-\Delta_{\ell} \sigma_{0}\right|}\left|\int_{\gamma}\left\|\mathcal{K}_{\rho+2}^{-1}(z) g\right\|_{B} \mathrm{~d} z\right| \\
& \leqslant\left.\frac{1}{2 \pi} \frac{C_{\mathrm{op}}}{\prod_{\ell=j}^{n}\left|1-\Delta_{\ell} \sigma_{0}\right|}\left|\int_{\gamma}\right| z\right|^{\mu-\rho-2} \mathrm{~d} z \mid\|g\|_{D} .
\end{aligned}
$$

Note that for $\rho>\mu-1$, the integral in (4.7) is bounded by a constant $C_{\sigma_{0}}$. The product can be estimated by means of Lemma A1 in the Appendix and the assertion follows.

The combination of the representation formula in Lemma 4.1 and the estimates in Lemma 4.3 directly leads to the following stability estimate of the discrete convolution.

THEOREM 4.4 Let Assumption 4.2 be satisfied and let $\Delta$ be sufficiently small that $1-\Delta \sigma_{0} \geqslant \alpha_{0}$ for some $\alpha_{0}>0$. Let $\rho$ be chosen according to (2.10) and assume that $g_{-1}^{(\rho)}=g_{0}^{(\rho)}=0$. Let $\phi_{n}$, for $1 \leqslant n \leqslant N$, denote the solution of (4.1). Then, there exists a constant $C$, depending only on $\sigma_{0}$ and $\alpha_{0}$, such that

$$
\left\|\phi_{n}\right\|_{B} \leqslant C \sum_{j=1}^{n}\left(\Delta_{j}+\Delta_{j-1}\right) e^{\delta_{0}\left(t_{n}-t_{j-1}\right)}\left\|\left[t_{j-2}, t_{j-1}, t_{j}\right] g^{(\rho)}\right\|_{D},
$$

with $\delta_{0}$ as in (4.6).

\subsection{Convergence}

By assuming that $g$ is $(\rho+2)$-times differentiable and has $(\rho+1)$ vanishing derivatives at the origin the exact solution can be written as follows:

$$
\phi\left(t_{n}\right)=\frac{1}{2 \pi \mathrm{i}} \int_{\gamma}\left(\mathcal{K}^{-1}\right)_{\rho+2}(\zeta) \mathrm{d} \zeta \int_{0}^{t_{n}} e^{\zeta\left(t_{n}-\tau\right)} g^{(\rho+2)}(\tau) \mathrm{d} \tau
$$

cf. (2.8). By interchanging the order of integration above, we obtain

$$
\phi\left(t_{n}\right)=\int_{0}^{t_{n}} Q^{(\rho+2, n)}(\tau) g^{(\rho+2)}(\tau) \mathrm{d} \tau
$$

with

$$
Q^{(k, n)}(\tau):=\frac{1}{2 \pi \mathrm{i}} \int_{\gamma}\left(\mathcal{K}^{-1}\right)_{k}(\zeta) e^{\zeta\left(t_{n}-\tau\right)} \mathrm{d} \zeta, \quad \text { for } k \in \mathbb{N} .
$$

TheOrem 4.5 Let Assumption 4.2 be satisfied and let $\Delta$ be sufficiently small that $1-\Delta \sigma_{0} \geqslant \alpha_{0}$ for some $\alpha_{0}>0$. Let $N \geqslant 1$ be the total number of time steps and let $\rho$ be chosen according to (2.10). 
We assume that the right-hand side in (4.1) satisfies $g \in C^{\rho+3}([0, T])$ and $g^{(\ell)}(0)=0$ for all $0 \leqslant \ell \leqslant$ $\rho+2$. We denote by $\phi_{n}$, for $1 \leqslant n \leqslant N$, the solution of (4.1). Then the following error estimate holds:

$$
\left\|\phi\left(t_{n}\right)-\phi_{n}\right\|_{B} \leqslant C \Delta c_{\rho-\mu}(\Delta)\left(\sum_{j=1}^{n} \frac{\Delta_{j}+\Delta_{j-1}}{2} e^{-\delta_{0} t_{j-1}} \max _{\substack{\tau \in\left[t_{j-2}, t_{j}\right] \\ \ell \in\{2,3\}}}\left\|g^{(\rho+\ell)}(\tau)\right\|_{D}\right)
$$

with

$$
c_{v}(\Delta)= \begin{cases}1+\log \frac{1}{\Delta} & \text { if } v=1 \\ 1 & \text { if } v>1\end{cases}
$$

Proof. The combination of (4.8) with (4.2) leads to the error representation

$$
\phi\left(t_{n}\right)-\phi_{n}=I_{1}+I_{2}+I_{3}
$$

where we set

$$
\begin{aligned}
& I_{1}:=\int_{0}^{t_{n}} Q^{(\rho+2, n)}(\tau) g^{(\rho+2)}(\tau) \mathrm{d} \tau-\sum_{j=1}^{n} \frac{\Delta_{j}+\Delta_{j-1}}{2} Q^{(\rho+2, n)}\left(t_{j-1}\right) g^{(\rho+2)}\left(t_{j-1}\right), \\
& I_{2}:=\sum_{j=1}^{n} \frac{\Delta_{j}+\Delta_{j-1}}{2}\left(Q^{(\rho+2, n)}\left(t_{j-1}\right)-Q_{j}^{(\rho+2, n)}\right) g^{(\rho+2)}\left(t_{j-1}\right), \\
& I_{3}:=\sum_{j=1}^{n} \frac{\Delta_{j}+\Delta_{j-1}}{2} Q_{j}^{(\rho+2, n)}\left(g^{(\rho+2)}\left(t_{j-1}\right)-2\left[t_{j-2}, t_{j-1}, t_{j}\right] g^{(\rho)}\right) .
\end{aligned}
$$

In the following, we will estimate these terms separately.

Estimate of $I_{2}$. For $I_{2}$, we start with

$$
Q^{(\rho+2, n)}\left(t_{j-1}\right)-Q_{j}^{(\rho+2, n)}=\frac{1}{2 \pi \mathrm{i}} \int_{\gamma}\left(\mathcal{K}^{-1}\right)_{\rho+2}(\zeta) d^{(j, n)}(\zeta) \mathrm{d} \zeta
$$

where

$$
d^{(j, n)}(\zeta):=e^{\zeta\left(t_{n}-t_{j-1}\right)}-\frac{1}{\prod_{\ell=j}^{n}\left(1-\Delta_{\ell} \zeta\right)}
$$

We split the contour $\gamma=\sigma_{0}+\mathrm{i} \mathbb{R}$ into

$$
\gamma^{\text {near }}:=\{\zeta \in \gamma:|\zeta \Delta|<\hat{C}\} \quad \text { and } \quad \gamma^{\text {far }}:=\gamma \backslash \gamma^{\text {near }}
$$

for some $\hat{C}=\mathcal{O}(1)$. From Lemma A1, we conclude

$$
\left|d^{(j, n)}(\zeta)\right| \leqslant 2 e^{\delta_{0}\left(t_{n}-t_{j-1}\right)}
$$


with $\delta_{0}$ as in (4.6). Hence, for the far-field part of the integral in (4.14), we obtain the estimation

$$
\begin{aligned}
\left\|\frac{1}{2 \pi \mathrm{i}} \int_{\gamma^{\mathrm{far}}}\left(\mathcal{K}^{-1}\right)_{\rho+2}(\zeta) d^{(j, n)}(\zeta) \mathrm{d} \zeta\right\|_{B \leftarrow D} & \leqslant C_{T} e^{-\delta_{0} t_{j-1}} \int_{c / \Delta}^{\infty} \frac{1}{\left(\sqrt{\sigma_{0}^{2}+\theta^{2}}\right)^{\rho+2-\mu}} \mathrm{d} \theta \\
& \leqslant C_{T} e^{-\delta_{0} t_{j-1}} \int_{c / \Delta}^{\infty} \frac{1}{\theta^{\rho+2-\mu}} \mathrm{d} \theta \leqslant C_{T} e^{-\delta_{0} t_{j-1}} \Delta^{\rho-(\mu-1)}
\end{aligned}
$$

where $c$ depends only on $\hat{C}$. Since $\rho \geqslant 1+\mu$, we conclude that $\Delta^{\rho-(\mu-1)} \leqslant \Delta^{2}$.

For the near-field part of the integral in (4.14), we conclude from Lemma A2 that

$$
\left|d_{n, j}(\zeta)\right| \leqslant C_{3} \Delta|\zeta|^{2} e^{-\delta_{0} t_{j-1}}
$$

and hence

$$
\left\|\frac{1}{2 \pi \mathrm{i}} \int_{\gamma^{\text {near }}}\left(\mathcal{K}^{-1}\right)_{\rho+2}(\zeta) d^{(j, n)}(\zeta) \mathrm{d} \zeta\right\|_{B \leftarrow D} \leqslant C \Delta e^{-\delta_{0} t_{j-1}} \int_{0}^{c / \Delta}|\zeta|^{\mu-\rho} \mathrm{d} \zeta .
$$

An easy calculation shows that

$$
\int_{0}^{c / \Delta}|\zeta|^{\mu-\rho} \mathrm{d} \zeta \leqslant C c_{\rho-\mu}(\Delta)
$$

with $c_{\rho-\mu}(\Delta)$ as in (4.10). Thus,

$$
\left\|\frac{1}{2 \pi \mathrm{i}} \int_{\gamma^{\text {near }}}\left(\mathcal{K}^{-1}\right)_{\rho+2}(\zeta) d^{(j, n)}(\zeta) \mathrm{d} \zeta\right\|_{B \leftarrow D} \leqslant C \Delta c_{\rho-\mu}(\Delta) e^{-\delta_{0} t_{j-1}} .
$$

In summary, we have proved

$$
\left\|I_{2}\right\|_{B} \leqslant C \Delta c_{\rho-\mu}(\Delta) \sum_{j=1}^{n} \frac{\Delta_{j}+\Delta_{j-1}}{2} e^{-\delta_{0} t_{j-1}}\left\|g^{(\rho+2)}\left(t_{j-1}\right)\right\|_{D}
$$

Estimate of $I_{1}$. Note that the difference $I_{1}$ is the error of the composite trapezoidal rule applied to the integral on the right-hand side in (4.12). We use only the fact that the trapezoidal rule is exact for constant functions to obtain the error estimate

$$
\left\|I_{1}\right\|_{B} \leqslant C \sum_{j=1}^{n} \Delta_{j}^{2} \max _{\tau \in\left[t_{j-1}, t_{j}\right]}\left\|\left(Q^{(\rho+2, n)} g^{(\rho+2)}\right)^{\prime}(\tau)\right\|_{B} .
$$

We obtain

$$
\left(\frac{\mathrm{d}}{\mathrm{d} \tau}\right)^{\ell} Q^{(\rho+2, n)}(\tau)=\frac{1}{2 \pi \mathrm{i}} \int_{\gamma}(-\zeta)^{\ell}\left(\mathcal{K}^{-1}\right)_{\rho+2}(\zeta) e^{\zeta\left(t_{n}-\tau\right)} \mathrm{d} \zeta
$$

This leads to the norm estimate for $\ell=0,1$,

$$
\max _{\tau \in\left[t_{j-1}, t_{j}\right]}\left\|\left(\frac{\mathrm{d}}{\mathrm{d} \tau}\right)^{\ell} Q^{(\rho+2, n)}(\tau)\right\|_{B \leftarrow D} \leqslant C e^{\sigma_{0}\left(t_{n}-t_{j-1}\right)} \int_{\gamma}|\zeta|^{\ell+\mu-(\rho+2)} \mathrm{d} \zeta \leqslant \tilde{C} e^{\sigma_{0}\left(t_{n}-t_{j-1}\right)},
$$


since $\ell+\mu-(\rho+2) \leqslant-2$. The Leibniz rule leads to

$$
\left\|I_{1}\right\|_{B} \leqslant C \Delta \sum_{j=1}^{n} \Delta_{j} e^{-\sigma_{0} t_{j-1}} \max _{\substack{\tau \in\left[t_{j-1}, t_{j}\right] \\ \ell \in\{2,3\}}}\left\|g^{(\rho+\ell)}(\tau)\right\|_{D}
$$

Estimate of $I_{3}$. A well-known property of divided differences is that there exists some $\tau \in\left[t_{j-2}, t_{j}\right]$ such that

$$
2\left[t_{j-2}, t_{j-1}, t_{j}\right] g^{(\rho)}=g^{(\rho+2)}(\tau),
$$

so that

$$
\left\|g^{(\rho+2)}\left(t_{j-1}\right)-g^{(\rho+2)}(\tau)\right\|_{D} \leqslant\left(\Delta_{j}+\Delta_{j-1}\right) \max _{\tau \in\left[t_{j-2}, t_{j}\right]}\left\|g^{(\rho+3)}(\tau)\right\|_{D}
$$

By means of Lemma 4.3 we finally obtain

$$
\left\|I_{3}\right\|_{B} \leqslant C \Delta \sum_{j=1}^{n} \frac{\Delta_{j}+\Delta_{j-1}}{2} e^{-\delta_{0} t_{j-1}} \max _{\tau \in\left[t_{j-2}, t_{j}\right]}\left\|g^{(\rho+3)}(\tau)\right\|_{D} .
$$

\section{Application to the wave equation}

Let $\Omega^{-} \subset \mathbb{R}^{3}$ be a bounded Lipschitz domain with boundary $\Gamma$. The unbounded complement is denoted by $\Omega^{+}:=\mathbb{R}^{3} \backslash \overline{\Omega^{-}}$. Let $\Omega \in\left\{\Omega^{-}, \Omega^{+}\right\}$. Our goal is to numerically solve the homogeneous wave equation

$$
\partial_{t}^{2} u=\Delta u \quad \text { in } \Omega \times(0, T),
$$

with initial conditions

$$
u(\cdot, 0)=\partial_{t} u(\cdot, 0)=0 \quad \text { in } \Omega
$$

and boundary conditions

$$
u=g \quad \text { on } \Gamma \times(0, T),
$$

on a time interval $(0, T)$ for some $T>0$ and given sufficiently smooth and compatible boundary data. For its solution, we employ an ansatz as a retarded single layer potential:

$$
\forall x \in \Omega, \quad \forall t \in(0, T), \quad u(x, t)=\int_{0}^{t} \int_{\Gamma} \frac{\delta(t-\|x-y\|)}{4 \pi\|x-y\|} \phi(y, \tau) \mathrm{d} \Gamma_{y} \mathrm{~d} \tau .
$$

The ansatz (5.2) satisfies the homogeneous equation (5.1a) and the initial conditions (5.1b). The extension $x \rightarrow \Gamma$ is continuous and hence, the unknown density $\phi$ in (5.2) is determined via the boundary conditions (5.1c), $u(x, t)=g(x, t)$. This results in the boundary integral equation for $\phi$,

$$
\forall t \in(0, T), \quad \int_{0}^{t} k(t-\tau) \phi(\tau) \mathrm{d} \tau=g(t) \quad \text { in } H^{1 / 2}(\Gamma),
$$

where $k(t): H^{-1 / 2}(\Gamma) \rightarrow H^{1 / 2}(\Gamma)$ is the kernel operator

$$
k(t) \phi=\int_{\Gamma} \frac{\delta(t-\|\cdot-y\|)}{4 \pi\|\cdot-y\|} \phi(y) \mathrm{d} \Gamma_{y},
$$


and $\delta(\cdot)$ denotes the Dirac delta distribution. The Sobolev space $H^{s}(\Gamma), s \geqslant 0$, is defined in the usual way (see, e.g., Hackbusch, 1992 or McLean, 2000). The range of $s$ for which $H^{s}(\Gamma)$ is defined may be limited, depending on the global smoothness of the surface $\Gamma$. Throughout, we let $[-k, k]$ denote the range of Sobolev indices for which $H^{s}(\Gamma)$ is defined, with the negative-order spaces defined by duality in the usual way. The norm is denoted by $\|\cdot\|_{H^{s}(\Gamma)}$.

Existence and uniqueness results for the solution of the continuous problem are proven in Bamberger \& Ha-Duong (1986), and Lubich (1994). The GCQ for (5.3) with the implicit Euler method then is given by

$$
\mathcal{K}_{-\rho}\left(\frac{1}{\Delta_{n}}\right) \phi_{n}=g_{n}^{(\rho)}-\sum_{j=1}^{n-1} \omega_{n, j}(0)\left(\left[\frac{1}{\Delta_{j}}, \frac{1}{\Delta_{j+1}}, \ldots, \frac{1}{\Delta_{n}}\right] \mathcal{K}_{-\rho}\right) \phi_{j} \quad \text { in } H^{1 / 2}(\Gamma),
$$

where the error analysis will justify the choice $\rho=3$.

Note that (5.4) is only semidiscrete since it is formulated in the infinite-dimensional space $H^{1 / 2}(\Gamma)$. The fully discrete space-time discretization will be introduced in Section 5.2.

\subsection{Analysis of the semidiscrete method}

The Laplace transformed integral operator is given by

$$
\mathcal{K}_{\rho}(\zeta) \phi:=\int_{\Gamma} \frac{e^{-\zeta\|\cdot-y\|}}{4 \pi \zeta^{\rho}\|\cdot-y\|} \phi(y) \mathrm{d} \Gamma_{y}
$$

It is well known (see Bamberger \& Ha-Duong, 1986, Proposition 3 and Lubich, 1994, Proposition 2.3) that $\mathcal{K}_{0}(\zeta): H^{-1 / 2}(\Gamma) \rightarrow H^{1 / 2}(\Gamma)$ is an isomorphism for all $\zeta$ with $\operatorname{Re} \zeta>0$ and also for $\zeta=0$. More precisely, the following continuity estimates hold.

Proposition 5.1 Let $\zeta \in \mathbb{C}$ with $\operatorname{Re} \zeta=\sigma_{0}>0$. Then

$$
\left\|\mathcal{K}_{0}(\zeta)\right\|_{H^{1 / 2}(\Gamma) \leftarrow H^{-1 / 2}(\Gamma)} \leqslant C \frac{1+\sigma_{0}^{2}}{\sigma_{0}^{3}}|\zeta|
$$

and

$$
\left\|\mathcal{K}_{0}^{-1}(\zeta)\right\|_{H^{-1 / 2}(\Gamma) \leftarrow H^{+1 / 2}(\Gamma)} \leqslant C \frac{1+\sigma_{0}}{\sigma_{0}}|\zeta|^{2}
$$

Proof. The first estimate follows from Bamberger \& Ha-Duong (1986, Proposition 3) and Lubich (1994, Proposition 2.3). Note that the second estimate is a consequence of the coercivity estimate (Bamberger \& Ha-Duong, 1986, Proposition 3)

$$
\operatorname{Re}\left(\zeta \mathcal{K}_{0}(\zeta) \psi, \psi\right)_{\Gamma} \geqslant c \frac{\min (1, \sigma)}{|\zeta|}\|\psi\|_{H^{-1 / 2}(\Gamma)}^{2} \quad \forall \psi \in H^{-1 / 2}(\Gamma), \quad \forall \zeta \in \mathbb{C} \text { with } \operatorname{Re} \zeta=\sigma>0
$$

where $(\cdot, \cdot)_{\Gamma}$ denotes the continuous extension of the $L^{2}(\Gamma)$ scalar product to the antilinear dual pairing $\langle\cdot, \cdot\rangle_{H^{1 / 2}(\Gamma) \times H^{-1 / 2}(\Gamma)}$.

Proposition 5.1 implies that Assumption 4.2 holds with $\mu=2$, so that Theorem 4.3 can be applied in the following form. 
THEOREM 5.2 We consider the GCQ (5.4) for the wave equation. Let $\Delta$ be sufficiently small that $1-\Delta \sigma_{0} \geqslant \alpha_{0}$ for some $\alpha_{0}>0$. Let $N \geqslant 1$ be the number of time steps and $\rho=3$. Let the boundary data in (5.1) satisfy $g \in C^{\rho+3}([0, T])$ and $g^{(\ell)}(0)=0$ for all $0 \leqslant \ell \leqslant \rho+2$. Let $\phi_{n}$, for $1 \leqslant n \leqslant N$, denote the solution of (4.1). Then the following error estimate holds:

$$
\left\|\phi\left(t_{n}\right)-\phi_{n}\right\|_{H^{-1 / 2}(\Gamma)} \leqslant C \Delta\left(1+\log \frac{1}{\Delta}\right)\left(\sum_{j=1}^{n} \frac{\Delta_{j}+\Delta_{j-1}}{2} e^{-\delta_{0} t_{j-1}} \max _{\substack{\tau \in\left[t_{j-2}, t_{j}\right] \\ \ell \in\{2,3\}}}\left\|g^{(\rho+\ell)}(\tau)\right\|_{H^{1 / 2}(\Gamma)}\right) .
$$

In the following, we will prove a shift theorem for the case that both the boundary $\Gamma$ of $\Omega^{-}$is analytic and the data $g(t)$ is analytic. The frequency variable is always assumed to be in a half plane (cf. (2.2))

$$
I_{\sigma_{0}}=\left\{z \in \mathbb{C}: \operatorname{Re} z \geqslant \sigma_{0}\right\} \quad \text { for some } \sigma_{0}>0 .
$$

Tubular neighbourhoods $\mathcal{N}$ of $\Gamma$ are open sets which satisfy

$$
\mathcal{N} \supset\left\{x \in \mathbb{R}^{d} \mid \operatorname{dist}(x, \Gamma)<\varepsilon\right\},
$$

for some $\varepsilon>0$.

Definition 5.3 For an open set $\mathcal{N} \subset \mathbb{R}^{3}$ and constants $C, \gamma>0$, we set

$$
\mathcal{A}(C, \gamma, \mathcal{N}):=\left\{f \in L^{2}(\mathcal{N}) \mid\left\|\nabla^{n} f\right\|_{L^{2}(\mathcal{N})} \leqslant C \gamma^{n} \max \{n+1,|\zeta|\}^{n} \quad \forall n \in \mathbb{N}_{0}\right\} .
$$

Here, $\left|\nabla^{n} u(x)\right|^{2}:=\sum_{\alpha \in \mathbb{N}_{0}^{3}:|\alpha|=n}(n ! / \alpha !)\left|D^{\alpha} u(x)\right|^{2}$.

For $s \in\{+,-\}$, we will need the standard trace operator $\gamma_{0}^{s}: H^{1}\left(\mathcal{N} \cap \Omega^{s}\right) \rightarrow H^{1 / 2}(\Gamma)$ and the onesided normal derivative $\gamma_{1}^{s}: H^{1}\left(\mathcal{N} \cap \Omega^{s}\right) \rightarrow H^{-1 / 2}(\Gamma)$, where the normal vector is oriented in both cases $\Omega^{+}, \Omega^{-}$into the unbounded domain $\Omega^{+}$.

Theorem 5.4 Assume that the boundary $\Gamma$ is analytic and star shaped. Let $g=\gamma_{0}^{-} G$ for some $G \in$ $\mathcal{A}\left(C_{G}, \gamma_{G}, \mathcal{N}_{G} \cap \Omega^{-}\right)$. For $\zeta \in \mathbb{C}$ with $\operatorname{Re} \zeta=\sigma_{0}>0$, let $\varphi=\mathcal{K}_{0}^{-1}(\zeta) g$. Then, $\varphi \in H^{q-1 / 2}(\Gamma)$ for any $q \geqslant 0$ and

$$
\|\varphi\|_{H^{q-1 / 2}(\Gamma)} \leqslant C_{q} C_{g} \begin{cases}|\zeta|^{2}, & q=0 \\ |\zeta|^{q+3 / 2}, & q \geqslant 1 / 2\end{cases}
$$

where

$$
C_{g}= \begin{cases}\|g\|_{H^{1 / 2}(\Gamma)}, & q=0, \\ C_{G}+\|g\|_{H^{1 / 2}(\Gamma)}, & q \geqslant 1 / 2 .\end{cases}
$$

Proof. Let $\zeta \in \mathbb{C}$ with $\operatorname{Re} \zeta=\sigma_{0}>0$. For $g$ as in the assumption of the theorem, let $\varphi=\mathcal{K}^{-1}(\zeta) g$. We define the potential

$$
u(x):=(\mathcal{S} \varphi)(x)=\int_{\Gamma} \frac{e^{-\zeta\|x-y\|}}{4 \pi\|x-y\|} \varphi(y) \mathrm{d} \Gamma_{y} \quad \forall x \in \mathbb{R}^{3} .
$$

Note that $u$ satisfies

$$
\begin{array}{rlrl}
-\Delta u+\zeta^{2} u & =0 & & \text { in } \Omega^{-} \cup \Omega^{+}, \\
u=g & & \text { on } \Gamma .
\end{array}
$$


From Sauter \& Schwab (2010, Lemma 3.1.9, Theorem 3.1.16), we conclude that $u \in H^{1}\left(\mathbb{R}^{d}\right)$. The wellknown jump relation is

$$
\left[\gamma_{0} u\right]_{\Gamma}=0 \text { and }\left[\gamma_{1} u\right]_{\Gamma}=-\varphi .
$$

From Melenk (2012, Theorem B.2), we conclude that the solution of (5.7) satisfies $u \in \mathcal{A}\left(C_{1}, \gamma, \mathcal{N} \cap\right.$ $\left.\Omega^{s}\right)$, where $C_{1} \leqslant C\left(C_{G}+|\zeta|^{-1}\|u\|_{1, \zeta \zeta \mid, \Omega^{s}}\right)$, where, for $\kappa>0$, the indexed norm $\|\cdot\|_{1, \kappa, \Omega}$ is given by

$$
\|f\|_{1, \kappa, \Omega}:=\sqrt{\|\nabla f\|_{L^{2}(\Omega)}^{2}+\kappa^{2}\|f\|_{L^{2}(\Omega)}^{2}} .
$$

From Melenk \& Sauter (2011, Remark 4.19), we conclude that

$$
\|u\|_{1,|\zeta|, \Omega^{s}} \leqslant C|\zeta|\left(|g|_{H^{1 / 2}(\Gamma)}+|\zeta|^{1 / 2}\|g\|_{L^{2}(\Gamma)}\right)
$$

so that

$$
\left\|\nabla^{n} u\right\|_{L^{2}(\mathcal{N})} \leqslant C\left(C_{G}+|g|_{H^{1 / 2}(\Gamma)}+|\zeta|^{1 / 2}\|g\|_{L^{2}(\Gamma)}\right) \gamma^{n} \max \{n+1,|\zeta|\}^{n} .
$$

By Green's formula we have

$$
\left\|\gamma_{1}^{s} u\right\|_{H^{-1 / 2}(\Gamma)}=\sup _{\psi \in H^{1 / 2}(\Gamma) \backslash\{0\}} \frac{\left|\left(\gamma_{1}^{s} u, \psi\right)_{\Gamma}\right|}{\|\psi\|_{H^{1 / 2}(\Gamma)}} \leqslant \sup _{\psi \in H^{1 / 2}(\Gamma) \backslash\{0\}} \frac{\|u\|_{1,|\zeta|, \Omega^{s}}\|Z \psi\|_{1,|\zeta|, \Omega^{s}}}{\|\psi\|_{H^{1 / 2}(\Gamma)}},
$$

where $(\cdot, \cdot)_{\Gamma}$ denotes the extension of the $L^{2}(\Gamma)$ scalar product to the antilinear dual pairing on $H^{-1 / 2}(\Gamma) \times H^{1 / 2}(\Gamma)$. From Bamberger \& Ha-Duong (1986, Lemma 1), we conclude that there exists an extension operator $Z: H^{1 / 2}(\Gamma) \rightarrow H^{1}\left(\Omega^{s}\right)$ such that

$$
\|Z \psi\|_{1,|\zeta|, \Omega^{s}} \leqslant C|\zeta|^{1 / 2}\|\psi\|_{H^{1 / 2}(\Gamma)} .
$$

Thus,

$$
\begin{aligned}
\|\varphi\|_{H^{-1 / 2}(\Gamma)} & \leqslant \sum_{s \in\{+,-\}}\left\|\gamma_{1}^{s} u\right\|_{H^{-1 / 2}(\Gamma)} \leqslant C|\zeta|^{1 / 2}\|u\|_{1,|\zeta|, \Omega^{+} \cup \Omega^{-}} \\
& \leqslant C|\zeta|^{3 / 2}\left(|g|_{H^{1 / 2}(\Gamma)}+|\zeta|^{1 / 2}\|g\|_{L^{2}(\Gamma)}\right) \leqslant C|\zeta|^{2}\|g\|_{H^{1 / 2}(\Gamma)} .
\end{aligned}
$$

For $q \geqslant 1 / 2$, from standard trace inequalities and (5.8) we obtain

$$
\begin{aligned}
\|\varphi\|_{H^{q-1 / 2}(\Gamma)} & \leqslant \sum_{s \in\{+,-\}}\left\|\gamma_{1}^{s} u\right\|_{H^{q-1 / 2}(\Gamma)} \\
& \leqslant C\|u\|_{H^{q+1}\left(\Omega^{+} \cup \Omega^{-}\right)} \leqslant C_{q}\left(C_{G}+\|g\|_{H^{1 / 2}(\Gamma)}\right)|\zeta|^{q+3 / 2} .
\end{aligned}
$$

\subsection{GCQ and spatial Galerkin discretization for RPIE}

We assume that $S_{\mathcal{G}}^{p}$ is a finite-dimensional boundary element space subordinate to a shape-regular surface mesh $\mathcal{G}=\left\{\tau_{i}: 1 \leqslant i \leqslant \tilde{M}\right\}$ of $\partial \Omega$, consisting of affine or possibly curved triangles (for the details we refer to Sauter \& Schwab, 2010, Chapter 4). 
The basis functions $b_{i}$ of the corresponding boundary element space

$$
S_{\mathcal{G}}^{p}=\operatorname{span}\left\{b_{i}: 1 \leqslant i \leqslant M\right\} \subset H^{-1 / 2}(\partial \Omega)
$$

are the usual Lagrange nodal basis functions, i.e., lifted piecewise polynomials of degree $p$ with local support. For brevity, we write $S$ for $S_{\mathcal{G}}^{p}$ if $\mathcal{G}$ and $p$ are clear from the context. The maximal mesh width is denoted by

$$
h_{\mathcal{G}}:=\max \left\{h_{\tau}: \tau \in \mathcal{G}\right\} \quad \text { with } h_{\tau}:=\operatorname{diam} \tau .
$$

The Galerkin discretization of the semidiscrete equation (5.4) is given by seeking functions $\phi_{n, S}$, $1 \leqslant n \leqslant N$, such that for all $\psi \in S$

$$
\left(\mathcal{K}_{-\rho}\left(\frac{1}{\Delta_{n}}\right) \phi_{n, S}, \psi\right)_{\Gamma}=\left(g_{n}^{(\rho)}, \psi\right)_{\Gamma}-\sum_{j=1}^{n-1} \omega_{n, j}(0)\left(\left(\left[\frac{1}{\Delta_{j}}, \frac{1}{\Delta_{j+1}}, \ldots, \frac{1}{\Delta_{n}}\right] \mathcal{K}_{-\rho}\right) \phi_{j, S}, \psi\right)_{\Gamma}
$$

where, again, $\rho=3$.

Let $P_{S}: H^{-1 / 2}(\Gamma) \rightarrow S$ be defined for all $\psi \in H^{-1 / 2}(\Gamma)$ by the relation

$$
\left(P_{S} \psi, \varphi\right)_{L^{2}(\Gamma)}=(\psi, \varphi)_{\Gamma} \quad \forall \varphi \in S
$$

The discrete Galerkin operator is given by

$$
\mathcal{K}_{-\rho, S}(\zeta):=P_{S} \mathcal{K}_{-\rho}(\zeta) P_{S}^{\star} \quad \text { with } P_{S}^{\star} \text { denoting the adjoint of } P_{S}
$$

Then, (5.10) can be written in operator form as

$$
\sum_{j=1}^{n} \omega_{n, j}(0)\left(\left[\frac{1}{\Delta_{j}}, \frac{1}{\Delta_{j+1}}, \ldots, \frac{1}{\Delta_{n}}\right] \mathcal{K}_{-\rho, S}\right) \phi_{j, S}=P_{S} g_{n}^{(\rho)}
$$

For the following, we need a generalization of Definition 5.3 to time-dependent functions.

Definition 5.5 For an open set $\mathcal{N} \subset \mathbb{R}^{3}$ and constants $C, \gamma, \sigma_{0}>0$ we set

$$
\begin{aligned}
\mathcal{A}\left(C, \gamma, \mathcal{N}, m, \sigma_{0}, T\right):= & \left\{f \in C^{m}\left([0, T], L^{2}(\mathcal{N})\right):\right. \\
& \left.\int_{0}^{\infty} e^{-\sigma_{0} \tau}\left\|\nabla^{n} f(\tau)\right\|_{L^{2}(\mathcal{N})} \mathrm{d} \tau \leqslant C \gamma^{n} \max \{n+1,|\zeta|\}^{n} \forall n \in \mathbb{N}_{0}\right\} .
\end{aligned}
$$

The subset of $\mathcal{A}\left(C, \gamma, \mathcal{N}, m, \sigma_{0}, T\right)$ with vanishing initial derivatives up to some order $k \leqslant m$ is given by

$$
\mathcal{A}_{0}\left(C, \gamma, \mathcal{N}, k, m, \sigma_{0}, T\right):=\left\{f \in \mathcal{A}\left(C, \gamma, \mathcal{N}, m, \sigma_{0}, T\right) \mid \forall 0 \leqslant \ell \leqslant k: f^{(\ell)}(0)=0\right\} .
$$

TheOREM 5.6 Assume that the boundary $\Gamma$ is analytic and star shaped. Let $g(t)=\gamma_{0} G(t)$ for some $G \in \mathcal{A}_{0}\left(C, \gamma, \mathcal{N}, p+7, p+8, \sigma_{0}, T\right)$ and we choose $\rho=3$ in (3.11), (5.10). Let $S_{\mathcal{G}}^{p}$ denote the boundary element space with mesh $\mathcal{G}$ and local polynomial degree $p$. Let $N \geqslant 1$ be the total number of time steps and $\Delta$ be sufficiently small that $1-\Delta \sigma_{0} \geqslant \alpha_{0}$ for some $\alpha_{0}>0$. Let $\phi_{n, S}$, for $1 \leqslant n \leqslant N$, denote the 
solution of (5.10). Then the following error estimate holds:

$$
\begin{aligned}
\left\|\phi\left(t_{n}\right)-\phi_{n, S}\right\|_{H^{-1 / 2}(\Gamma)} \leqslant & C_{p} C_{g} h_{\mathcal{G}}^{p+3 / 2}+C \Delta\left(1+\log \frac{1}{\Delta}\right) \\
& \times\left(\sum_{j=1}^{n} \frac{\Delta_{j}+\Delta_{j-1}}{2} e^{-\delta_{0} t_{j-1}} \max _{\substack{\tau \in\left[t_{j-2}, t_{j}\right] \\
\ell \in\{2,3\}}}\left\|g^{(\rho+\ell)}(\tau)\right\|_{H^{1 / 2}(\Gamma)}\right),
\end{aligned}
$$

with $C_{g}$ given by (5.6).

Proof. Lemma 4.1 can be applied and we obtain

$$
\phi_{n, S}=\sum_{j=1}^{n}\left(\Delta_{j}+\Delta_{j-1}\right) Q_{j, S}^{(\rho+2, n)}\left[t_{j-2}, t_{j-1}, t_{j}\right] P_{S} g_{j}^{(\rho)},
$$

where

$$
Q_{j, S}^{(k, n)}:=\frac{1}{2 \pi \mathrm{i}} \int_{\gamma} \frac{\left(\mathcal{K}_{S}^{-1}\right)_{k}(z)}{\prod_{\ell=j}^{n}\left(1-\Delta_{\ell} z\right)} \mathrm{d} z \quad \forall 1 \leqslant j \leqslant n+1 .
$$

To estimate $\phi_{n, S}-\phi\left(t_{n}\right)$, we write

$$
\phi\left(t_{n}\right)-\phi_{n, S}=I_{0}+I_{1}+I_{2}+I_{3},
$$

where

$$
I_{0}:=\int_{0}^{\infty}\left(Q^{(\rho+2, n)}(\tau) g^{(\rho+2)}(\tau)-Q_{S}^{(\rho+2, n)}(\tau) P_{S} g^{(\rho+2)}(\tau)\right) \mathrm{d} \tau
$$

with

$$
Q_{S}^{(k, n)}(\tau):=\frac{1}{2 \pi \mathrm{i}} \int_{\gamma}\left(\mathcal{K}_{S}^{-1}\right)_{k}(\zeta) e^{\zeta\left(t_{n}-\tau\right)} \mathrm{d} \zeta
$$

$I_{1}, I_{2}, I_{3}$ are defined as in (4.12), where $Q^{(\rho+2, n)}(\tau)$ has to be replaced by $Q_{S}^{(\rho+2, n)}(\tau)$ and $Q_{j}^{(\rho+2, n)}$ by $Q_{j, S}^{(\rho+2, n)}$.

Estimate of $I_{0}$ : We apply partial integration $(p+5)$-times and use $g^{(\ell)}(0)=0$ for all $\ell \leqslant \rho+p+6$ to obtain

$$
I_{0}=\int_{0}^{\infty}\left(Q^{(\rho+p+7, n)}(\tau) g^{(\rho+p+7)}(\tau)-Q_{S}^{(\rho+p+7, n)}(\tau) P_{S} g^{(\rho+p+7)}(\tau)\right) \mathrm{d} \tau .
$$

Let $\psi:=\left(\mathcal{K}^{-1}\right)_{\rho+p+7}(z) g^{(\rho+p+7)}$ and $\psi_{S}:=\left(\mathcal{K}_{S}^{-1}\right)_{\rho+p+7}(z) P_{S} g^{(\rho+p+7)}$. The $H^{-1 / 2}(\Gamma)$-orthogonal projection onto $S$ is denoted by $\Pi_{S}: H^{-1 / 2}(\Gamma) \rightarrow S$. Then

$$
\psi-\psi_{S}=\left(\psi-\Pi_{S} \psi\right)+\left(\Pi_{S} \psi-\psi_{S}\right)
$$

By applying $\left(\mathcal{K}_{S}\right)_{-\rho}(z)$ to the second term and using Galerkin's orthogonality, we obtain

$$
\left(\mathcal{K}_{S}\right)_{-\rho}(z)\left(\Pi_{S} \psi-\psi_{S}\right)=P_{S} \mathcal{K}_{-\rho}(z)\left(\Pi_{S} \psi-\psi\right)
$$


Since the coercivity estimate (5.5) holds for all $\varphi \in S$, we conclude that ${ }^{4}\left(\mathcal{K}_{S}\right)_{-\rho}(z) P_{S}$ exists and satisfies the same bound as the continuous operator

$$
\left\|\left(\mathcal{K}_{S}^{-1}\right)_{\rho}(z) P_{S}\right\|_{H^{-1 / 2}(\Gamma) \leftarrow H^{+1 / 2}(\Gamma)} \leqslant C \frac{1+\sigma_{0}}{\sigma_{0}}|z|^{2-\rho} .
$$

Using this estimate and Proposition 5.1 we obtain

$$
\left\|\Pi_{S} \psi-\psi_{S}\right\|_{H^{-1 / 2}(\Gamma)} \leqslant C|z|^{2-\rho}\left\|\mathcal{K}_{-\rho}(z)\left(\left(\Pi_{S} \psi-\psi\right)\right)\right\|_{H^{1 / 2}(\Gamma)} \leqslant \tilde{C}|z|^{3}\left\|\Pi_{S} \psi-\psi\right\|_{H^{-1 / 2}(\Gamma)} .
$$

In total, we have proved that

$$
\left\|\psi-\psi_{S}\right\|_{H^{-1 / 2}(\Gamma)} \leqslant \tilde{C}\left(1+|z|^{3}\right)\left\|\Pi_{S} \psi-\psi\right\|_{H^{-1 / 2}(\Gamma)} .
$$

Hence,

$$
\left\|I_{0}\right\|_{H^{-1 / 2}(\Gamma)} \leqslant \tilde{C}_{T} \frac{1}{2 \pi} \int_{0}^{\infty} e^{-\sigma_{0} \tau}\left|\int_{\gamma}\left(1+|\zeta|^{3}\right)\left\|\left(\Pi_{S}-I\right)\left(\mathcal{K}^{-1}\right)_{\rho+p+7}(\zeta) g^{(\rho+p+7)}(\tau)\right\|_{H^{-1 / 2}(\Gamma)} \mathrm{d} \zeta\right| \mathrm{d} \tau .
$$

Standard approximation properties of the space $S_{\mathcal{G}}^{p}$ lead to

$$
\left\|\left(\Pi_{S}-I\right)\left(\mathcal{K}^{-1}\right)_{\rho+p+7}(\zeta) g^{(\rho+p+7)}(\tau)\right\|_{H^{-1 / 2}(\Gamma)} \leqslant C h_{\mathcal{G}}^{p+3 / 2}\left\|\left(\mathcal{K}^{-1}\right)_{\rho+p+7}(\zeta) g^{(\rho+p+7)}(\tau)\right\|_{H^{p+1}(\Gamma)} .
$$

We apply Theorem 5.4 to obtain

$$
\int_{0}^{\infty} e^{-\sigma_{0} \tau}\left(1+|\zeta|^{3}\right)\left\|\left(\mathcal{K}^{-1}\right)_{\rho+p+7}(\zeta) g^{(\rho+p+7)}(\tau)\right\|_{H^{p+1}(\Gamma)} \leqslant C_{p} C_{g}|\zeta|^{-2}
$$

The integral over $\zeta$ is bounded so that we obtain the required bound for $I_{0}$.

The estimates of $I_{1}, I_{2}, I_{3}$ are just a repetition of the arguments in the proof of Theorem 4.5 since also the Galerkin operator satisfies $\left\|\mathcal{K}_{S}^{-1}(\zeta) P_{S}\right\| \leqslant C|\zeta|^{2}$.

\section{Conclusion}

In this paper, we have developed a GCQ method with variable time stepping for solving one-sided convolution equations. As in the original convolution quadrature, the continuous equation is transformed to the Laplace domain and the transformed solution can be characterized as the solution of an ODE. In contrast to the original method, we introduce variable time stepping for the solution of the ODE. The discrete equation is transformed back to the time domain resulting in the GCQ method. The operators involved in this equation can be computed as Newton's divided differences applied to the transfer operator. Although the convolution structure is not inherited at the discrete level, we expect that for problems with nonuniformly distributed irregularities in the right-hand side, the savings in the number of time steps by adaptive time stepping can be very significant. Future work will be devoted to the development of a fast GCQ.

We have developed a new theory for the analysis of the GCQ which is different from the theory of the original convolution quadrature. The reason is that the discrete equation is no longer a proper discrete convolution and, hence, cannot be transformed to the Fourier-Laplace domain by the discrete

\footnotetext{
${ }^{4}$ Note that $\left(\mathcal{K}^{-1}\right)_{m, S}(\zeta)=\zeta^{-m} \mathcal{K}_{0, S}^{-1}(\zeta)=\left(\mathcal{K}_{-m, S}(\zeta)\right)^{-1}$
} 
Fourier transform. Instead, we have developed direct estimates for Newton's divided differences of the transfer operator which allows us to stay on the 'time-domain side'. As an important application of this theory, we consider the formulation of the wave equation in unbounded domains as RPIE and prove that the GCQ converges at an optimal rate (up to a logarithmic term).

In Lopez-Fernandez \& Sauter (2012b) and Lopez-Fernandez \& Sauter (2012a), we developed a fast and stable algorithmic version of the GCQ and reported the results of numerical experiments which illustrate the advantage of variable time stepping for some important classes of applications.

\section{Funding}

This work was partially supported by the Spanish grant MTM 2010-19510 (M.L.-F.).

\section{Acknowledgement}

Part of this work was carried out during a visit of the second author to the Department of Mathematics, University of California, San Diego, La Jolla. This support is gratefully acknowledged.

\section{REFERENCES}

Bamberger, A. \& Ha-Duong, T. (1986) Formulation variationnelle espace-temps pour le calcul par potientiel retardé de la diffraction d'une onde acoustique. Math. Meth. Appl. Sci., 8, 405-435.

DE Boor, C. (2005) Divided differences. Surv. Approx. Theory, 1, 46-69.

Haсквusch, W. (1992) Elliptic Differential Equations. Berlin: Springer.

Lopez-Fernandez, M., Lubich, C. \& SchäDle, A. (2008) Adaptive, fast, and oblivious convolution in evolution equations with memory. SIAM J. Sci. Comput., 30, 1015-1037.

Lopez-Fernandez, M. \& SAuter, S. A. (2012a) Fast and stable contour integration for high order divided differences via elliptic functions. Technical Report 08-2012. Zürich: Institut für Mathematik.

Lopez-Fernandez, M. \& Sauter, S. A. (2012b) A generalized convolution quadrature with variable time stepping. Part II: algorithms and numerical results. Technical Report 09-2012. Zürich: Institut für Mathematik.

Lubich, C. (1988a) Convolution quadrature and discretized operational calculus I. Numer. Math., 52, 129-145.

Lubich, C. (1988b) Convolution quadrature and discretized operational calculus II. Numer. Math., 52, 413-425.

LuBICH, C. (1994) On the multistep time discretization of linear initial-boundary value problems and their boundary integral equations. Numer. Math., 67, 365-389.

Lubich, C. (2004) Convolution quadrature revisited. BIT Numer. Math., 44, 503-514.

Lubich, C. \& Ostermann, A. (1993) Runge-Kutta methods for parabolic equations and convolution quadrature. Math. Comp., 60, 105-131.

McLean, W. (2000) Strongly Elliptic Systems and Boundary Integral Equations. Cambridge: Cambridge University Press.

Melenk, J. (2012) Mapping properties of combined field Helmholtz boundary integral operators. SIAM J. Math. Anal., 44, 2599-2636.

Melenk, J. M. \& SAUter, S. A. (2011) Wave-number explicit convergence analysis for Galerkin discretizations of the Helmholtz equation. SIAM J. Numer. Anal., 49, 1210-1243.

SAUter, S. \& Schwab, C. (2010) Boundary Element Methods. Heidelberg: Springer.

SAUter, S. \& Veit, A. (2012) A Galerkin method for retarded boundary integral equations with smooth and compactly supported temporal basis functions. Numer. Math., (electronic first) DOI 10.1007/s00211-012-0483-7. 


\section{Appendix A. Estimate of $d^{(j, n)}$}

In this appendix, we will derive some estimates for the function $d^{(j, n)}$ as in (4.15).

Lemma A1 Let $\gamma=\sigma_{0}+\mathrm{i} \mathbb{R}$ for some $\sigma_{0}>0$. For the maximal mesh width $\Delta$, we assume $1-\sigma_{0} \Delta>0$. For all $\zeta \in \gamma$, it holds that

$$
\frac{1}{\prod_{\ell=j}^{n}\left|1-\Delta_{\ell} \zeta\right|} \leqslant e^{\delta_{0}\left(t_{n}-t_{j-1}\right)} \quad \text { with } \delta_{0}:=\frac{\sigma_{0}}{1-\sigma_{0} \Delta} .
$$

Proof. The assertion follows from

$$
\frac{1}{\prod_{\ell=j}^{n}\left|1-\Delta_{\ell} \zeta\right|} \leqslant \frac{1}{\prod_{\ell=j}^{n}\left|1-\sigma_{0} \Delta_{\ell}\right|}=\exp \left\{\sum_{\ell=j}^{n} \log \left(1+\frac{\Delta_{\ell} \sigma_{0}}{1-\Delta_{\ell} \sigma_{0}}\right)\right\} \leqslant e^{\delta_{0}\left(t_{n}-t_{j-1}\right)} .
$$

Next, we will derive an estimate for $d_{n, j}$ for all $\zeta \in \gamma^{\text {near }}$; cf. (4.16).

Lemma A2 Let $\gamma=\sigma_{0}+\mathrm{i} \mathbb{R}$ for some $\sigma_{0}>0$ and let $\gamma^{\text {near }}$ be as in (4.16). For the maximal mesh width $\Delta$, we assume $1-\sigma_{0} \Delta>0$. Then, for all $\zeta \in \gamma^{\text {near }}$ it holds that

$$
\left|d_{n, j}(\zeta)\right| \leqslant C_{3} \min \left\{1, \Delta|\zeta|^{2}\right\} e^{-\delta_{0} t_{j-1}},
$$

where $C_{3}$ depends on the final time $T$ and the parameter $\sigma_{0}$.

Proof. Let

$$
c_{\ell}:=\frac{1}{1-\zeta \Delta_{\ell}}
$$

and observe $\left|c_{\ell}\right| \geqslant\left(1-\sigma_{0} \Delta\right)^{-1}>0$. Furthermore, let

$$
\varepsilon_{\ell}:=e^{\Delta_{\ell} \zeta}-\frac{1}{1-\zeta \Delta_{\ell}} \quad \text { and } \quad \varepsilon:=\left(\varepsilon_{\ell}\right)_{\ell=j}^{n} .
$$

Then, it is easy to see that there is a constant $C_{2}$ depending only on $C$ such that

$$
\left|\varepsilon_{\ell}\right| \leqslant C_{2}\left|\zeta \Delta_{\ell}\right|^{2} \quad \forall \zeta \in I_{\sigma_{0}, \Delta}
$$

We write

$$
d_{n, j}(\zeta)=\prod_{\ell=j}^{n}\left(c_{\ell}+\varepsilon_{\ell}\right)-\prod_{\ell=j}^{n} c_{\ell}
$$

Taylor expansion of the function $g_{n, j}(\varepsilon):=\prod_{\ell=j}^{n}\left(c_{\ell}+\varepsilon_{\ell}\right)$ about $\varepsilon=0$ yields

$$
g_{n, j}(\varepsilon)=g_{n, j}(0)+\left\langle\nabla g_{n, j}(\theta \varepsilon), \varepsilon\right\rangle
$$


for some $\theta \in[0,1]$. For the derivatives of $g_{n, j}$, we obtain

$$
\frac{\partial g_{n, j}}{\partial \varepsilon_{k}}(\theta \varepsilon)=\prod_{\substack{\ell=j \\ \ell \neq k}}^{n}\left(c_{\ell}+\theta \varepsilon_{\ell}\right)
$$

From

$$
\left|\prod_{\substack{\ell=j \\ \ell \neq k}}^{n}\left(c_{\ell}+\theta \varepsilon_{\ell}\right)\right|=\left|\prod_{\substack{\ell=j \\ \ell \neq k}}^{n}\left(\frac{1-\theta}{1-\zeta \Delta_{\ell}}+\theta e^{\Delta_{\ell} \zeta}\right)\right|
$$

and

$$
\left|\frac{1}{1-\Delta_{\ell} \zeta}\right| \leqslant \frac{1}{1-\Delta_{\ell} \sigma_{0}} \leqslant e^{\delta_{0} \Delta_{\ell}} \quad \text { with } \delta_{0}:=\frac{\sigma_{0}}{1-\Delta \sigma_{0}},
$$

we obtain

$$
\left|\prod_{\substack{\ell=j \\ \ell \neq k}}^{n}\left(c_{\ell}+\theta \varepsilon_{\ell}\right)\right| \leqslant\left|\prod_{\substack{\ell=j \\ \ell \neq k}}^{n}\left((1-\theta) e^{\delta_{0} \Delta_{\ell}}+\theta e^{\Delta_{\ell} \delta_{0}}\right)\right| \leqslant \prod_{\substack{\ell=j \\ \ell \neq k}}^{n} e^{\Delta_{\ell} \delta_{0}} \leqslant e^{\delta_{0}\left(t_{n}-t_{j-1}\right)} .
$$

From (A.1), we derive

$$
\begin{aligned}
\left|\left\langle\nabla g_{n, j}(\theta \varepsilon), \varepsilon\right\rangle\right| & \leqslant C_{2} \sum_{\ell=j}^{n} e^{\delta_{0}\left(t_{n}-t_{j-1}\right)}\left|\zeta \Delta_{\ell}\right|^{2} \\
& \leqslant C_{2} \Delta|\zeta|^{2}\left|t_{n}-t_{j-1}\right| e^{\delta_{0}\left(t_{n}-t_{j-1}\right)} \\
& \leqslant\left(C_{2} T e^{\delta_{0} T}\right) \Delta|\zeta|^{2} e^{-\delta_{0} t_{j-1}}
\end{aligned}
$$

and the estimate

$$
\left|d_{n, j}(\zeta)\right| \leqslant C_{3} \Delta|\zeta|^{2} e^{-\delta_{0} t_{j-1}}
$$

follows. The other estimate directly follows from Lemma A1:

$$
\left|d_{n, j}(\zeta)\right| \leqslant\left|e^{\zeta\left(t_{n}-\tau_{j-1}\right)}\right|+\frac{1}{\prod_{\ell=j}^{n}\left|1-\Delta_{\ell} \zeta\right|} \leqslant C_{T} e^{-\delta_{0} t_{j-1}} .
$$

The combination of these two estimates leads to the assertion. 